\section{Acute rhinosinusitis and systemic corticosteroids}

I read with interest the $C M A J$ article by Venekamp and colleagues, ${ }^{1}$ but I disagree with the authors' findings that suggest systemic corticosteroid monotherapy had no clinically important benefit in acute rhinosinusitis. The proportion of patients with resolution of facial pain or pressure on day 7 was $62.5 \%$ in the prednisolone group and $55.8 \%$ in the placebo group (absolute risk difference $6.7 \%$ ). Studies with possible clinical importance have $95 \%$ confidence intervals (CIs) that include the value of the minimal clinically important difference (MCID) and a MCID greater than the point estimate of the efficacy. ${ }^{2}$ Based on this, the $95 \%$ CI $(-7.9 \%$ to $21.2 \%)$ obtained for the point estimate included both the point estimate $(6.7 \%)$ and the MCID used for the study (20\%).

Also, the proportion of patients with resolution of severe facial pain or pressure on day 7 was significantly higher among those receiving prednisolone compared with those receiving placebo (absolute risk difference 10.6\%, 95\% CI $1.0 \%$ to $20.2 \%$ ). Furthermore in Table 2, which shows the proportion of patients with resolution of symptoms on day 7 , the prednisolone group shows a tendency toward an overall beneficial effect compared with placebo.

One reason why the full effect of systemic corticosteroids cannot be elicited from the study is lack of patient selection. The Infectious Diseases Society of America guidelines distinguish clinically between acute bacterial/viral rhinosinusitis and acute rhinosinusitis due to other causes based on illness pattern and duration. ${ }^{3}$ There was no attempt to identify patients in the sample who might benefit from other treatment approaches. It is possible that the short-term benefits are higher among those with acute rhinosinusitis due to other causes compared with patients with acute bacterial rhinosinusitis who might require antibiotic therapy. ${ }^{3}$ A previous guideline suggests that systemic corticosteroids be reserved for those with nasal polyps, severe nasal swelling due to inflammation of the mucous membrane, or for whom other treatment approaches have failed. ${ }^{4}$

I thank the authors for the study, but I believe the clinical importance of systemic corticosteroids for treating acute rhinosinusitis should not completely be discarded until we identify the subgroups of patients who will benefit from them.

\section{Kingsley N. Ukwaja}

Resident

Department of Internal Medicine, Federal

Teaching Hospital, Abakaliki, Ebonyi

State, Nigeria

\section{References}

1. Venekamp RP, Bonten MJM, Rovers MM, et al. Systemic corticosteroid monotherapy for clinically diagnosed acute rhinosinusitis: a randomized controlled trial. CMAJ 2012;184:E751-7.

2. Man-Son-Hing M, Laupacis A, O'Rourke K, et al. Determination of the clinical importance of study results: a review. J Gen Intern Med 2002;17:469-76.

3. Rosenfeld RM, Andes D, Bhattacharyya N, et al. Clinical practice guideline: adult sinusitis. Otolaryngol Head Neck Surg 2007;137(Suppl):1-31.

4. Slavin RG, Spector SL, Bernstein IL, et al. The diagnosis and management of sinusitis: a practice parameter update. J Allergy Clin Immunol 2005;116(Suppl):13-47.

CMAJ 2013. DOI:10.1503/cmaj.113-2092

\section{The author responds}

We sincerely thank Dr. Ukwaja ${ }^{1}$ for his commentary on our CMAJ article. ${ }^{2}$ To the best of our knowledge, no sign, symptom or test has been identified that can accurately differentiate viral from bacterial infection in patients with clinically diagnosed acute rhinosinusitis. Clinical practice guidelines' recommendations to differentiate between viral and bacterial acute rhinosinusitis based on illness pattern and/or duration of symptoms are mostly based on consensus rather than scientific evidence. To enhance the generalizability of our trial findings, we included the broad population of patients with clinically diagnosed acute rhinosinusitis encountered in primary care. ${ }^{2}$

Both our primary and secondary outcomes revealed small but clinically unimportant differences between the systemic corticosteroid and the placebo group. We are therefore confident that our main conclusion "lack of effect of systemic corticosteroids in patients with clinically diagnosed acute rhinosinusitis" is justified. However, we agree with Ukwaja's final statement that there may be a subgroup of patients who do benefit from systemic corticosteroids. The magnitude of the effect size found in our study is in agreement with the effect sizes reported in previous trials on antibiotics in acute rhinosinusitis. ${ }^{3}$ There might be a subgroup of patients that could benefit from antibiotics and an individual patient-data metaanalysis has been performed. ${ }^{3}$ Unfortunately, no clinical sign or symptom could be detected to predict beneficial effects of antibiotics. Finding subgroups of patients who really benefit from antibiotics is challenging, and it is likely that this will also be the case for detecting subgroups who will benefit from systemic corticosteroids. Future research is needed to identify those subgroups that do benefit from either antibiotics or corticosteroids. Until then, we recommend refraining from these treatment options in patients with uncomplicated clinically diagnosed acute rhinosinusitis, because symptoms are self-limiting in the majority of patients within 2 to 4 weeks.

\section{Roderick P. Venekamp MD PhD (on behalf of all authors)}

The Julius Center for Health Sciences and Primary Care, and the Department of Otorhinolaryngology, University Medical Center Utrecht, Utrecht, the Netherlands

\section{References}

1. Ukwaja KN. Acute rhinosinusitis and systemic corticosteroids [letter]. CMAJ 2013;185:62.

2. Venekamp RP, Bonten MJM, Rovers MM, et al. Systemic corticosteroid monotherapy for clinically diagnosed acute rhinosinusitis: a randomized controlled trial. CMAJ 2012;184:E751-7.

3. Young J, De SA, Merenstein D, et al. Antibiotics for adults with clinically diagnosed acute rhinosinusitis: a meta-analysis of individual patient data. Lancet 2008;371:908-14.

\section{CMAJ 2013. DOI:10.1503/cmaj.113-2093}

\section{Antimicrobials in farming}

I wish to applaud Barbara Sibbald for her editorial on antimicrobial use in agriculture and its implications for human health. ${ }^{1}$ I was interested to learn about the degree to which Canada lags behind other countries in regulating the agricultural use of antimicrobials.

I have 2 comments on the editorial: With respect to the article's statement that 